\title{
Drug therapy for atrial fibrillation: quo vadis?
}

\section{Ronald M. Jardine}

Cardiologist in private practice, Linmed Hospital, Benoni

Address for correspondence:

Dr Ronald M. Jardine

PO Box 99

Benoni

1500

South Africa

Email:

e-mail: jardinerm@gmail.com

ABSTRACT Atrial fibrillation has always been the most common sustained cardiac arrhythmia, and its incidence is increasing worldwide. Despite evolving ablation techniques, the vastness of the number of cases entrenches drug therapy as the mainstay of treatment for the majority of cases both now and in the foreseeable future.

Drug therapy for atrial fibrillation includes drugs for ventricular rate control, anti-coagulation, and cardioversion/ maintenance of sinus rhythm (rhythm control). This review summarizes the available data on new drugs in each of these 3 areas.

In the area of rhythm control, it is clear that primary prevention of atrial fibrillation is achieved by a number of drugs in common clinical usage in hypertension, heart failure, and vascular disease, viz. blockers of the reninangiotensin system and statins. Primary prevention is also promising with novel therapies such as anti-inflammatory therapy, pirfenidone, and $\Omega-3$ poly-unsaturated fatty acids.

Secondary prevention with anti-arrhythmic drugs producing multiple channel blockade is proven to be efficacious, and atrial-selective anti-arrhythmic drugs are an attractive development and will avoid ventricular pro-arrhythmia.

A number of new drugs with novel mechanisms of action have mostly not yet undergone clinical trials, but are discussed here, and include gap junction modulators, stretch-activated channel blockers, sodium-calcium exchange inhibitors and new ion channel blockers.

SAHeart 2008; 5:142-147

\section{INTRODUCTION}

This year is the centenary of the first ECG recording of atrial fibrillation (AF) by Hering, (I) but drug therapy for the condition was reported long before that in 1835 by Bouillaud. ${ }^{(2)}$ Current drug therapy for AF includes drugs for ventricular rate control, anti-arrhythmic drugs for rhythm control, and anti-coagulants. The 3 essential problems of this arrhythmia are symptoms, thrombo-embolism, and tachycardia-induced cardiomyopathy. The prevention of tachycardia-induced cardiomyopathy requires rate control; the prevention of thrombo-embolism requires anti-coagulation; and the suppression of symptoms requires rate control in all patients and rhythm control in some. Those are patients who have symptoms despite rate control or in whom rhythm control is suspected to be a better strategy. Rhythm control is suspected to be a better strategy in younger patients, when symptoms are severe, in the presence of diastolic dysfunction in hypertension or hypertrophic cardiomyopathy, and in mitral stenosis.

\section{RATE CONTROL}

Current options for rate control include the well-known drugs digoxin rate-slowing calcium channel blockers, viz. verapamil and diltiazem, beta-blockers, and amiodarone, and the "ablate and pace" procedure. Digoxin, although historically the very first drug used in this condition is known to be rather ineffective, especially when exercise heart rates are evaluated, and there is also concern about increased mortality with digoxin in females with heart failure (Digoxin Investigation Group Trial) ${ }^{(3)}$ especially if the serum level is $>1.2 \mathrm{ng} / \mathrm{ml},{ }^{(4)}$ and in both genders in the SPORTIF III and $V$ trials in patients with AF.(5) Amiodarone, although efficacious in ventricular rate control, is seldom used for this indication alone because of serious non-cardiac toxicity.

What are future options for rate control? Dronederone is an amiodarone congener which is non-iodinated and consequently has fewer side-effects, especially thyroid and pulmonary, and consequently may well have a place in the future rate control armamentarium. It has been shown to lower the ventricular rate by $12 \mathrm{bpm}$ at rest and $24 \mathrm{bpm}$ on exercise in the ERATO trial. ${ }^{(6)} \mathrm{A}$ number of adenosine agonists 
specific for the AI receptor are in development and include intravenous (tecadenoson) and oral (seladenoson) forms. Direct injection of the AV node is feasible because of its sub-endocardial location and animal experiments have shown that cell therapy by injection of fibroblasts in this way is possible and that this retards AV conduction. A novel gene therapy was reported whereby an adenovirus carrying a transgene, namely the alpha subunit of the inhibitory $G$ protein, was injected down the AV nodal artery, and resulted in suppression of calcium channel activity, and significant slowing of the ventricular response even after adrenaline infusion in a porcine model..$^{(7)}$

\section{ANTI-COAGULATION}

Current options for anti-coagulation in AF consist only of warfarin and aspirin. The latest ACC / AHA / ESC guidelines for AF management $(2006)^{(8)}$ stratify the risk for thrombo-embolism according to certain "major", "moderate" and "minor" risk factors (Table I). Warfarin is

TABLE I: Risk factors for thrombo-embolism in atrial fibrillation

\begin{tabular}{|l|l|l|}
\hline Major & Moderate & Minor \\
\hline Previous thrombo-embolism & Congestive heart failure & Female gender \\
\hline Mitral stenosis & Hypertension & Age $65-75$ years \\
\hline Prosthetic heart valve & Age $>75$ years & Coronary artery disease \\
\hline & Diabetes & Echo: left atrial $($ LA) size \\
\hline Ejection fraction $<0.35$ & and LA appendage flow \\
\hline & Thrombophilia \\
\hline & Estrogen therapy \\
\hline
\end{tabular}

TABLE II: Future options for anti-coagulation in AF

\begin{tabular}{|l|l|}
\hline Alternative to aspirin & Alternative to warfarin \\
\hline Clopidogrel & Anti-platelet \\
& Clopidogrel \\
\hline Indirect factor Xa inhibitors \\
Idraparinux \\
\hline Biotinylated idraparinux \\
\hline Direct factor Xa inhibitors \\
LY5I77|7 \\
YMI50 \\
DUI76b \\
Apixaban \\
Betrixaban \\
Rivaroxaban \\
\hline Direct thrombin inhibitors \\
Ximelagatran \\
Dabigatran \\
\hline
\end{tabular}

recommended if any one major risk factor is present, or if two moderate risk factors are present, or if one moderate and a number of minor risk factors are present. Low-dose aspirin is recommended for the rest.

New options for anti-coagulation are listed in Table II. Clopidogrel as an alternative to aspirin is the subject of an ongoing trial namely ACTIVE A. Clopidogrel plus aspirin as an alternative to warfarin has proved to be inferior in the ACTIVE W trial. (9) Recently idraparinux in comparison to warfarin proved to be more effective at prevention of thrombo-embolism, but caused significantly more bleeding (AMADEUS trial). ${ }^{(10)}$ A number of direct factor $X a$ inhibitors are in clinical trials at present including apixaban (ARISTOTLE and AVERROES), betrixaban (EXPERT) and rivaroxaban (ROCKET-AF). Although the first direct thrombin inhibitor ximelagatran has been withdrawn from further investigation because of liver toxicity, dabigatran is the subject of the ongoing RELY trial which should be reported in the next 2 years. All of these trials use warfarin as the comparator. ${ }^{(1)}$

\section{RHYTHM CONTROL}

Apart from radio-frequency ablation options for rhythm control (pulmonary veins, accessory pathway, and cavo-tricuspid isthmus for flutter) in selected patients, the vast majority of patients with AF in whom the rhythm control strategy is being pursued, will require drug therapy. Currently in South Africa there are 4 drugs available for this purpose, viz. the 2 class IC drugs flecainide and propafenone, a betablocker with class III activity sotalol, and a multiple class action drug amiodarone. The clinical niches and usage of these drugs are discussed elsewhere and will not be elaborated on here. The 6 so-called "strategy trials", viz. PIAF,(12) STAF,(13) RACE,(14) AFFIRM,(15) HOT CAFÉ(16) and AF-CHF(17) concluded that rhythm control is not superior to rate control, so that it might well be questioned whether there is a point in new rhythm control drugs. It was however clear from a subsequent analysis of the largest of these trials, viz. AFFIRM that sinus rhythm was a very powerful predictor of survival (hazard ratio 0.54), but that this was offset by a powerful negative effect of the drugs used to control rhythm (hazard ratio 1.4I). ${ }^{(18)}$ Thus rhythm-controlling drugs without the adverse effects of the current generation are likely to have a survival benefit.

Future options for rhythm control are listed in Table III. Upstream therapy, also known as "primary prevention" refers to therapies which 
TABLE III: Future options for rhythm control in AF

\begin{tabular}{|c|c|}
\hline Upstream therapy & Other \\
\hline Renin-angiotensin system blockade & Gap junction / connexin modulators \\
\hline Statins & Stretch - activated channel blockers \\
\hline Anti-inflammatory therapy & $\mathrm{Na} / \mathrm{Ca}$ exchange inhibitors \\
\hline Anti-fibrotic therapy & New I $I_{N_{a}}$ current blockers \\
\hline Omega-3 poly-unsaturated fatty acids & $I_{\text {Kach }}$ channel blockers \\
\hline PPAR- $\gamma$ modulators & $\mathrm{I}_{\mathrm{Ks}}$ channel blockers \\
\hline Anti-oxidants & Heat shock protein blocker \\
\hline Multiple channel blockade & Gene therapy \\
\hline Tedisamil & Stem cell therapy \\
\hline $\begin{array}{r}\text { Amiodarone congeners - dronedarone } \\
\text { celivarone }\end{array}$ & \\
\hline \multicolumn{2}{|l|}{ Atrial selectivity } \\
\hline Atrial repolarization-delaying agents & \\
\hline ATI-2042 (amiodarone congener) & \\
\hline Serotonin $5 \mathrm{HT}_{4}$ receptor antagonists & \\
\hline
\end{tabular}

modulate the electro-anatomical remodelling of substrate for atrial fibrillation. Benefits have been shown for prevention of atrial fibrillation by all 4 classes of blockers of the renin-angiotensin system, viz. ß-blockers, angiotensin converting enzyme(ACE)-inhibitors, angiotensin receptor blockers (ARBs) and aldosterone antagonists. A recent metaanalysis showed that ß-blockers in systolic heart failure resulted in a $27 \%$ risk reduction for new-onset $A F^{(19)}$ To what extent this is a direct anti-arrhythmic effect versus an indirect effect on blood pressure, pump function and prevention of myocardial infarction, is not clear. ACEinhibitors were first shown in a small Dutch study with lisinopril to have a benefit in the maintenance of sinus rhythm after cardioversion;(20) subsequently in a large post-MI trial with trandolopril there was a benefit in AF prevention;(21) and in a large heart failure trial with enalapril (SOLVD) there was a similar benefit. (22) In the case of ARBs, in a secondary prevention trial, Madrid from Madrid showed that irbesartan combined with amiodarone was better at maintenance of sinus rhythm after cardioversion than amiodarone alone. ${ }^{(23)}$ Subsequently primary prevention of AF has been shown in large trials in hypertension with losartan (LIFE), ${ }^{(24)}$ and in heart failure with valsartan (VAL-HeFT) ${ }^{(25)}$ and candesartan (CHARM). ${ }^{(26)}$ Recently a meta-analysis of prevention of AF with ACE-inhibitors and ARBs reported both to be effective provided that there was either LV hypertrophy or systolic dysfunction. ${ }^{(27)}$

Statins have been mooted to have an anti-arrhythmic benefit, particularly for atrial fibrillation, on the basis of experimental and observational clinical studies. This effect may be due to their antiinflammatory or anti-oxidant properties, and they are known to have effects on matrix metallo-proteinases, nitric oxide synthesis and L-type calcium currents which may also translate into anti-arrhythmic benefit. Earlier this year a meta-analysis of 6 studies and 3557 patients showed a significant benefit, more marked in the 3 secondary prevention trials than in the 3 primary prevention trials (which were in patients having acute coronary syndrome or undergoing cardiac surgery). ${ }^{(28)}$

The background to anti-inflammatory therapy is that $66 \%$ of atrial biopsies in lone AF have myocarditis, and C-reactive protein (CRP) levels are raised in AF, more so in permanent AF than in persistent AF and least so in paroxysmal AF. The success of cardioversion has been related to CRP levels and thrombo-embolic risk correlates with CRP levels. A study has shown that treatment with methylprednisolone successfully prevents recurrence and the development of permanence in atrial fibrillation, and that this also correlated with reduction in CRP levels. ${ }^{(29)}$

Atrial fibrosis in structural heart disease is an important substrate for AF. Anti-fibrosis therapy includes the aldosterone antagonists spironolactone and eplerenone, and a specific anti-fibrotic drug pirfenidone. In a dog study, pirfenidone has been shown to significantly reduce vulnerability to AF and atrial remodeling. ${ }^{(30)}$

The superiority of amiodarone as an anti-fibrillatory drug has been attributed to its mode of action, which involves multiple channel blockade. Some newer drugs also have this property and include congeners of amiodarone and tedisamil (Figure I). Tedisamil is a blocker of $I_{\text {Kr }}, I_{K s}, I_{\text {to }}, I_{K A T P}, I_{N a}, I_{K u r}$ and in addition is a gap junction modulator (vide infra). The oral formulation has been abandoned because it causes diarrhea but the intravenous form is effective for chemical cardioversion of $A F$, with a half life of $8-13$ hours so that early recurrence of atrial fibrillation is rare. The mean time to cardioversion is 35 minutes and the success rate is about $55 \%$ in AF but only about half that in atrial flutter. The drug does prolong QT interval which resulted in ventricular tachycardia in 2 of 53 patients in the reported study. $(31)$

Dronedarone, like amiodarone, blocks $I_{\mathrm{Kr}}, I_{\mathrm{Ks}}, I_{\mathrm{Ca} a}, I_{\mathrm{to}}, I_{\mathrm{Na}}, I_{\mathrm{Kach}}$, alpha and beta adrenergic receptors, but contains no iodine and, as a result, has fewer side-effects especially thyroid and pulmonary. A number of clinical trials have been completed with this drug. DAFNE was a dose-ranging study comparing 400mg versus 600mg versus $800 \mathrm{mg}$ BD and showed that $600 \mathrm{mg}$ and $800 \mathrm{mg}$ were no more effective, but caused more side-effects. ${ }^{(32)}$ ANDROMEDA was a trial in heart 
failure with an ejection fraction of $<0.35$, and was terminated prematurely in January 2003 because of an increased mortality of $8.1 \%$ with dronedarone compared to $3.8 \%$ on placebo, due to worsening heart failure. It is thought that a rise in serum creatinine from a renal tubular effect of the drug led to discontinuation of ACE-I/ARB therapy by clinicians. ${ }^{(33)}$ EURIDIS and ADONIS were identical trials conducted in Europe and America showing the efficacy of dronedarone at the maintenance of sinus rhythm after cardioversion with significant success (hazard ratio $=0.75$ and $\mathrm{P}<0.00 \mathrm{I}$ ) after I year follow-up. ${ }^{(34)}$ The oneyear recurrence rate on dronedarone was $65 \%$, whereas it was only $25 \%$ with amiodarone in the Canadian trial of Atrial Fibrillation, ${ }^{(35)}$ suggesting that it is not quite as efficacious as amiodarone. In ATHENA, the biggest anti-arrhythmic drug trial to date with 4628 patients, dronedarone was shown to significantly reduce the primary endpoint, which was death or time to first hospitalization for a cardiovascular reason, with a hazard ratio of 0.76 and a $P<0.001$. Most of the benefit was in the reduction of hospitalization but cardiovascular deaths were also reduced significantly. A rise in creatinine occurred in $4.7 \%$ of patients on dronedarone vs. $1 \%$ on placebo. ${ }^{(36)}$ Celivarone is another amiodarone congener which is non-iodinated with a completed dose ranging trial (MAIA) and a completed trial on oral chemical cardioversion (CORYFREE) not reported yet.
The attractive property of atrial selectivity is being pursued by a number of pharmaceutical investigators with a number of as yet unnamed molecules known as "atrial repolarization-delaying agents" such as AVE 0II8, S9947, S2095I, ASD7009, NIP-I4I/2 and XEND0I01/2. The most advanced information concerns RSDI235 now called vernakalant, which blocks the $I_{\text {Kur }}$ channel which is only found in the atria so that the drug has minimal effect on ventricular repolarization. It is also however a blocker of $\mathbb{K}_{\text {Ach, }}$ Ito, and frequency and voltage dependent $I_{\mathrm{Na}^{\prime}}$ so that this and the other drugs in this class are not completely atrial selective. It exhibits use dependency and has minimal hemodynamic effects. The intravenous form has been tested in a doseranging phase 2 trial (CRAFT) as well as a series of phase 3 clinical trials (ACT I-IV), showing a $50 \%$ success rate in chemical cardioversion of short-duration $A F$ in a median time of I I minutes, but it is ineffective against atrial flutter and only $27 \%$ effective in heart failure. No torsades de pointes has been seen. Sneezing, taste disturbance and nausea are side-effects. ${ }^{(37)}$ An oral formulation for maintenance of sinus rhythm after cardioversion shows promise in phase 2 trials. ${ }^{(38)}$

Serotonin infusion is known to cause sinus tachycardia, atrial tachycardia and AF. Consequently antagonists of serotonin and specifically the $5 \mathrm{HT} 4$ receptor, which is only found in the atria and not the ventricles, have been developed, viz. RS-1003002 and SB-207266 (now called

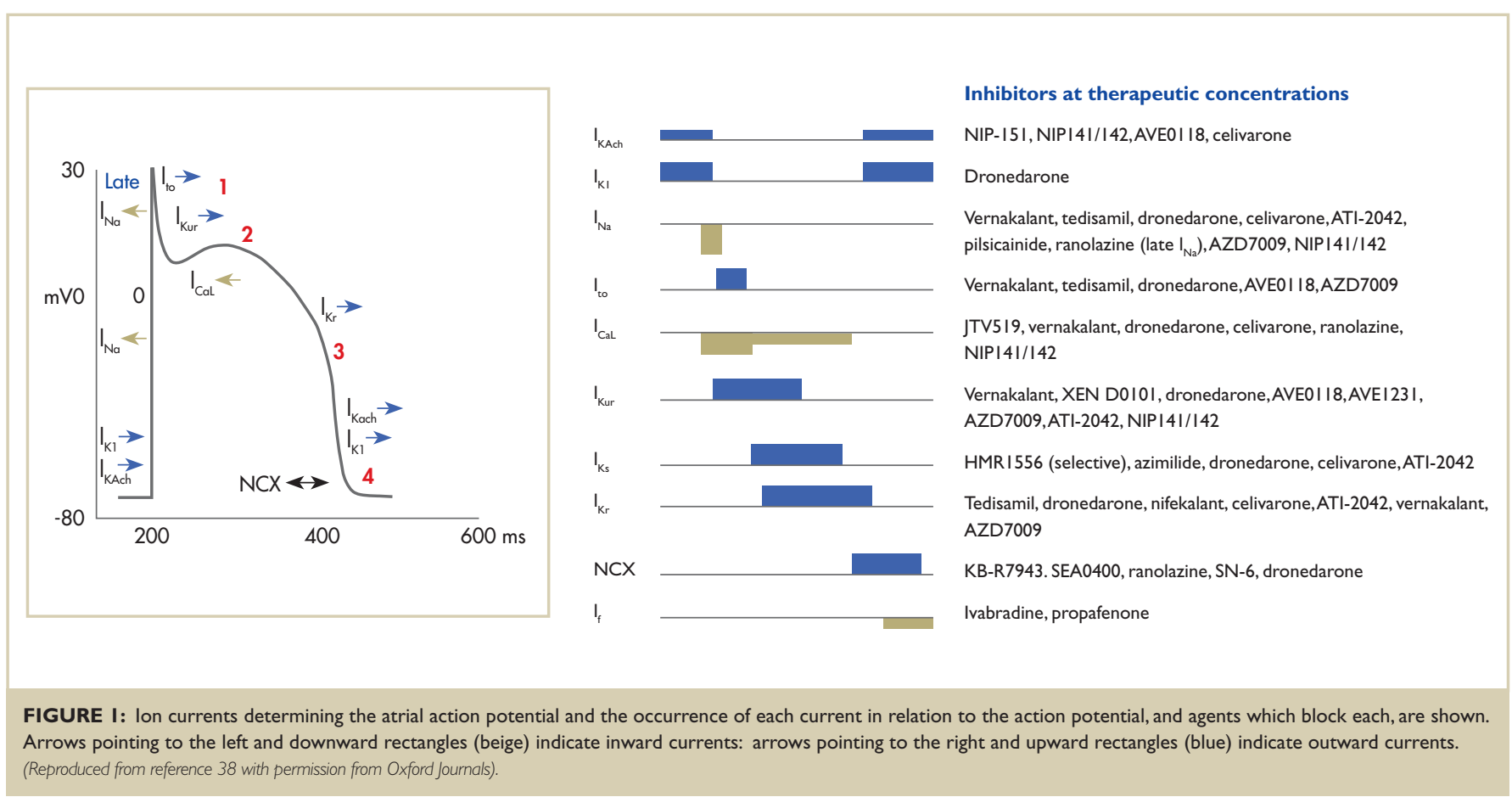


piboserod). In a study piboserod has proved to be ineffective at maintaining sinus rhythm after cardioversion.

The final group of new drugs for rhythm control is a miscellaneous one, only sharing the fact that they have novel mechanisms of action. Gap junctions are physiologically important for rapid cell-to-cell conduction and are composed of connexons, which in turn are made up of 6 connexins, numbers 40,43 and 45 being found in the atrial myocardium. Some previously mentioned drugs, viz. losartan and tedisamil facilitate conduction through gap junctions, thereby increasing conduction velocity and shortening the effective refractory period. Interest is focused on specific gap junction modulators, and in particular GAP486 / ZPI23, now called rotigaptide. This is a hexapeptide which augments gap junction conductance and improves cell-to-cell coupling. In dog experiments, rotigaptide reduces vulnerability to AF in a mitral regurgitation model but not in a heart failure model, ${ }^{(39)}$ and in atrial ischemia, but not in an atrial tachycardia model. ${ }^{(40)}$

Atrial dilatation may activate certain ion channels, and blockade of these channels by stretch activated channel blockers might be therapeutically useful. Substances known to have this property include gadolinium, amiloride, cationic antibiotics, and poly-unsaturated fatty acids. A selective blocker of these channels exists in the venom of the Chilean Rose tarantula spider, a 35-amino acid peptide known as GsMTx-4

The Na-Ca exchange mechanism on myocyte membranes normally moves 3 molecules of $\mathrm{Na}$ into the cell for every one molecule of $\mathrm{Ca}$ moving out of the cell. In rapid atrial pacing or fibrillation, this exchange is reversed. Inhibitors of $\mathrm{Na}-\mathrm{Ca}$ exchange prevent this reversal. An example is KB-R7943 which blocks a number of other channels also.

Ranolazine is a piperazine derivative with anti-anginal / anti-ischemic properties and was investigated in the MERLIN-TIMI 36 trial where it was shown to significantly reduce unsustained ventricular tachycardia and supra-ventricular tachycardia incidence, and it also reduced the incidence of new AF, albeit not significantly (I.7\% vs. $2.4 \%, P=0.08){ }^{(4)}$ Ranolazine uniquely is an inhibitor of the late phase of the inward sodium current (late $\mathrm{I}_{\mathrm{Na}}$ ) but is also a blocker of multiple other channels including peak $\left.\left.I_{\mathrm{Na}^{\prime}}\right|_{\mathrm{Kr}_{r}}\right|_{\mathrm{Ks}}, \mathrm{I}_{\mathrm{CaL}}$ and $\mathrm{Na}-\mathrm{Ca}$ exchange. It causes minimal QTc prolongation (2-6mS).

\section{CONCLUSION}

Although a strategy of ventricular rate control may not be inferior to rhythm control in some patients with AF, drug treatment for rhythm control is likely to increase in the future as a result both of the increasing incidence of atrial fibrillation as well as the development of exciting new drugs which will not have adverse effects, especially pro-arrhythmia. Advances in ablation techniques for AF will have an impact only on a small segment of the patient population. Hopefully new anti-coagulants will be unburdened from the need for frequent laboratory testing in the future 


\section{REFERENCES:}

I. Hering HE. Das electrocardiogram des pulsus irregularis perpetuus. Dtsch Arch 1908:94:205

2. Bouillaud JB. Traite clinique des maladies du couer. Paris. JB Bailliere 1835

3. Rathore SS, Wang Y and Krumholtz HM. Sex-based differences in the effect of digoxin for the treatment of heart failure. N Engl J Med 2002;347: I 403-I I.

4. Adams KF, Patterson JH, Gattis WA, et al. Relationship of serum digoxin concentration to mortality and morbidity in women in the Digoxin Investigation Group Trial. J Am Coll Cardiol 2005:46:497-504.

5. Gjesdal K, Feyzi J and Olsson B. Digitalis: a dangerous drug in atrial fibrillation? An analysis of the SPORTIF III and V data. Heart 2008;94:191-6.

6. Davy JM, et al. ESC congress abstract. Eur Heart J 2006;27:885.

7. Donahue JK, Heldman AW, Fraser $\mathrm{H}$, et al. Focal modification of electrical conduction in the heart by viral gene transfer. Nat Med 2000;6: I 395-8.

8. ACC / AHA / ESC Guidelines for the management of patients with atrial fibrillation executive summary. Eur Heart J 2006;27:1 979-2030.

9. ACTIVE Investigators. Clopidogrel plus aspirin vs. oral anti-coagulation for atrial fibrillation in the Atrial Fibrillation Clopidogrel Trial with Irbesartan for prevention of Vascular Events (ACTIVEW): A randomized controlled trial. Lancet 2006;367:1903-12.

10. AMADEUS Investigators. Comparison of idraparinux with vitamin $\mathrm{K}$ antagonists for prevention of thrombo-embolism in patients with atrial fibrillation: a randomized, openlabel, non-inferiority trial. Lancet 2008;371:315-21.

11. Turpie AGG. New oral anti-coagulants in atrial fibrillation. Eur Heart J 2008;29: I55-65.

12. Hohnloser SH, Kuck K-H, Lilienthal J. Rhythm or rate control in atrial fibrillation Pharmacologic Intervention in Atrial Fibrillation (PIAF): a randomized trial. Lancet 2000;356: 1789-94

13. Carlsson J, Miketic S, Windeler J, et al. Randomized trial of rate-control versus rhythmcontrol in persistent atrial fibrillation: the Strategies of Treatment of Atrial Fibrillation (STAF) study. J Am Coll Cardiol 2003;41:1690-6.

14. Van Gelder IC, Hagens VE, Bosker HA, et al. A comparison of rate control and rhythm control in patients with recurrent persistent atrial fibrillation. $N$ Engl J Med 2002;347:1834-40.

15. AFFIRM investigators. A comparison of rate control and rhythm control in patients with atrial fibrillation. N Engl J Med 2002;347: I 825-33.

16. Opolski G, Torbicki A, Kosior DA, et al. Rate control versus rhythm control in patients with non-valvular atrial fibrillation: the results of the Polish How to Treat Chronic Atrial Fibrillation (HOT CAFÉ) study. Chest 2004;126:476-86.

17. Roy D, Talajic M, Nattel S, et al. Rhythm control versus rate control for atrial fibrillation and heart failure. N Engl J Med 2008;358:2667-77.

18. AFFIRM investigators. Relationship between sinus rhythm, treatment, and survival in the Atrial Fibrillation Follow-up Investigation of Rhythm Management (AFFIRM) study. Circulation 2004; 109:1509-13.

19. Nasr IA, Bouzamondo A, Hulot HS, et al. Prevention of atrial fibrillation onset by betablocker treatment in heart failure: a meta-analysis. Eur Heart J 2007;28:457-62.

20. Van der Berg MP, Crijns H,Van Veldhuisen DJ, et al. Effects of lisinopril in patients with heart failure and chronic atrial fibrillation. J Cardiac Failure 1995; I (5):355-63.

21. Pedersen OD, Bagger $\mathrm{H}$, Kober $\mathrm{L}$, et al. Trandolapril reduces the incidence of atrial fibrillation after acute myocardial infarction in patients with left ventricular dysfunction. Circulation 1999; 100(4):376-80

22. Vermes E, Tardif JC, Bourassa MG, et al. Enalapril decreases the incidence of atrial fibrillation in patients with left ventricular dysfunction. Circulation 2003; 107(23):2926-31.

23. Madrid AH, Bueno MG, Rebollo JM, et al. Use of irbesartan to maintain sinus rhythm in patients with long-lasting persistent atrial fibrillation: a prospective and randomized study. Circulation 2002; 106:331-6.

24. Wachtell K, Lehto M, Gerdts E, et al. Angiotensin II receptor blockade reduces newonset atrial fibrillation and subsequent stroke compared to atenolol. J Am Coll Cardiol 2005;45:712-9.

25. Maggioni AP, Latini R, Carson PE, et al.Valsartan reduces the incidence of atrial fibrillation in patients with heart failure: results from the Valsartan Heart Failure Trial (Val-HeFT). Am Heart J 2005; 1 49(3):548-57.
26. Ducharme A, Swedberg K, Pfeffer MA, et al. Prevention of atrial fibrillation in patients with symptomatic chronic heart failure by candesartan in the Candesartan in Heart failure: Assessment of Reduction in Mortality and morbidity (CHARM) program. Am Heart J 2006; I 52(I):86-92.

27. Healey JS, Baranchuk A, Crystal E, et al. Prevention of atrial fibrillation with angiotensinconverting enzyme inhibitors and angiotensin receptor blockers - a meta-analysis. J Am Coll Cardiol 2005;45: 1832-9.

28. Fauchier L, Pierre B, De Labriolle A, et al. Anti-arrhythmic effect of statin therapy and atrial fibrillation - a meta-analysis of randomized controlled trials. J Am Coll Cardiol 2008:5।:828-35.

29. Dernellis J and Panaretou M. Relationship between C-reactive protein concentrations during glucocorticoid therapy and recurrent atrial fibrillation. Eur Heart J 2004;25: I l 00-7.

30. Lee KW, Everett TH, Rahmutula D, et al. Pirfenidone prevents the development of a vulnerable substrate for atrial fibrillation in a canine model of heart failure. Circulation 2006; 1 14:1703-12.

31. Hohnloser SH, Dorian P, Straub M, et al. Safety and efficacy of intravenously administered tedisamil for rapid conversion of recent-onset atrial fibrillation or atrial flutter. J Am Coll Cardiol 2004:44:99-104

32. Touboul P, Brugada J, Capucci A, et al. Dronedarone for prevention of atrial fibrillation: a dose-ranging study. Eur Heart J 2003;24:1481-7.

33. Kober L, Torp-Pedersen C, McMurray JJV, et al. Increased mortality after dronedarone therapy for severe heart failure. N Engl J Med 2008;358:2678-87.

34. Singh BN, Connolly SJ, Crijns HJGM, et al. Dronedarone for maintenance of sinus rhythm in atrial fibrillation or flutter. N Engl J Med 2007;357:987-99.

35. Roy D, Talajic M, Dorian P, et al. Amiodarone to prevent recurrence of atrial fibrillation. N Engl J Med 2000;342:9। 3-20

36. Hohnloser $\mathrm{SH}$. Effects of dronedarone on cardiovascular outcomes in high-risk patients with atrial fibrillation or atrial flutter - results of the ATHENA trial. HRS 2008. Oral presentation

37. Roy D, Pratt CM,Torp-Pedersen C, et al.Vernakalant hydrochloride for rapid conversion of atrial fibrillation - a phase 3, randomized, placebo-controlled trial. Circulation 2008 1 17:1518-25

38. Savelieva I and Camm J. Anti-arrhythmic drug therapy for atrial fibrillation: current anti-arrhythmic drugs, investigational agents, and innovative approaches. Europace 2008; 10:647-65.

39. Guerra JM, Everett TH, Lee KW, et al. Effects of the gap junction modifier rotigaptide (ZPI23) on atrial conduction and vulnerability to atrial fibrillation. Circulation 2006; 1 14:110-8.

40. Shiroshita-Takeshita A, Sakabe M, Haugan K, et al. Model-dependent effects of the gap junction conduction-enhancing anti-arrhythmic peptide rotigaptide (ZPI23) on experimental atrial fibrillation in dogs. Circulation 2007; 1 15:31 0-8.

41. Scirica BM, Morrow DA, Hod H, et al. Effect of ranolazine, an anti-anginal agent with novel electrophysiological properties, on the incidence of arrhythmias in patients with non-ST segment-elevation acute coronary syndrome. Circulation 2007; I 1 6: 1647-52. 